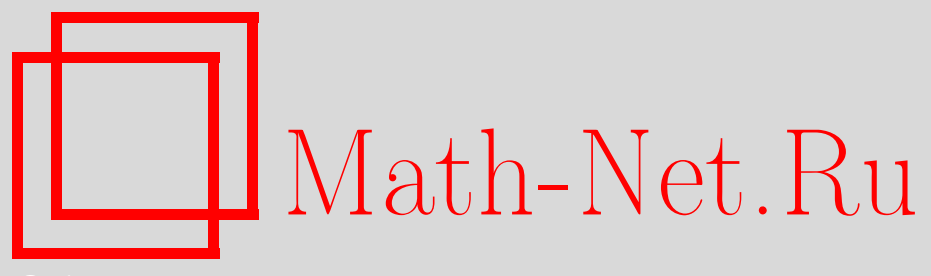

А. Энсисо, Д. Пералта-Салас, Классическая и квантовая интегрируемость гамильтонианов без состояний рассеяния, $Т M \Phi, ~ 2006$, том 148, номер 2, 249-268

DOI: https://doi.org/10.4213/tmf2084

Использование Общероссийского математического портала Math-Net.Ru подразумевает, что вы прочитали и согласны с пользовательским соглашением http://www . mathnet.ru/rus/agreement

Параметры загрузки:

IP : 3.85 .73 .92

26 апреля 2023 г., $17: 40: 48$

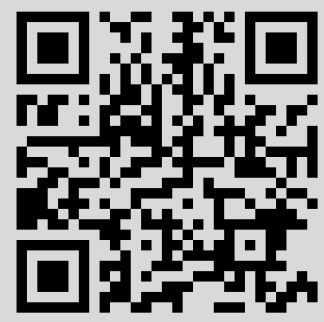




\title{
КЛАССИЧЕСКАЯ И КВАНТОВАЯ ИНТЕГРИРУЕМОСТЬ ГАМИЛЬТОНИАНОВ БЕЗ СОСТОЯНИЙ РАССЕЯНИЯ
}

\begin{abstract}
Установлено, что каждый квантовый гамильтониан без состояний рассеяния обладает полным семейством сохраняющихся величин независимо от размерности системы. На основании этого результата произведено сравнение общих свойств классических и квантовых интегрируемых систем. Обсуждаются несколько подходящих примеров и доказано утверждение, касающееся статистического распределения энергий. В качестве полезного дополнительного результата получено еще одно подтверждение гипотезы Берри-Табора без привлечения квазиклассического предела.
\end{abstract}

Ключевые слова: квантовая механика, интегрируемость, спектральная теория, гипотеза Берри-Табора.

\section{1. ВВЕДЕНИЕ И ОПРЕДЕЛЕНИЯ}

Интегрируемость всегда играла важную роль в качественных исследованиях классических и квантовых динамических систем. В квантовом случае имеются два основных подхода к этому вопросу, каждый из которых соответствует различным областям физики. В математической физике обычно имеют дело с конкретными квантовыми моделями (с возможной зависимостью от свободных параметров) и пытаются явно вычислить первые интегралы гамильтонианов с помощью теории групп [1]. В случае квантового хаоса часто представляют интерес спектральные свойства, характеризующие хаотические или интегрируемые гамильтонианы, а модели, для которых точная информация является недоступной, обычно исследуются с помощью квазиклассического приближения и численных расчетов [2].

В настоящей работе, пытаясь соединить обе точки зрения, мы получаем некоторую точную информацию о квантовой интегрируемости путем наложения условий на спектр гамильтониана, а не путем ограничения исследования анализом конкретных моделей. Полученные результаты приводят также к проясняющему сравнению

${ }^{*}$ Depto. de Física Teórica II, Universidad Complutense, Madrid, Spain. E-mail: aenciso@fis.ucm.es, dperalta@fis.ucm.es 
классической и квантовой механик и к точному исследованию степени общности статистики Пуассона для гамильтонианов без состояний рассеяния.

Классический гамильтониан $H(\mathbf{x}, \mathbf{p})$ представляет собой функцию из $2 n$-мерного фазового пространства в пространство вещественных чисел. Концепция классической интегрируемости впервые была точно сформулирована Лиувиллем, который определил, что классический гамильтониан $H(\mathbf{x}, \mathbf{p})$ является интегрируемым, когда он обладает $n$ функционально независимыми первыми интегралами, находящимися в инволюции и обладающими некоторой степенью регулярности, т.е. являющимися гладкими или аналитическими. Сложность динамики, определяемой гамильтонианом $H(\mathbf{x}, \mathbf{p})$, т.е. регулярное или хаотическое поведение орбит гамильтониана, сильно зависит от его интегрируемости. В частности, динамика не рассматривается как хаотическая, если гамильтониан $H(\mathbf{x}, \mathbf{p})$ является интегрируемым.

Квантовый гамильтониан $H$ представляет собой самосопряженный оператор, действующий в сепарабельном гильбертовом пространстве $\mathcal{H}$. Наивное определение квантовой интегрируемости, которое встречается во многих научных статьях, вытекает из определения классической интегрируемости. А именно квантовый гамильтониан $H$ является интегрируемым в наивном смысле, если существуют $n$ независимых линейных операторов $T_{i}(i=1, \ldots, n$, где $n$ - размерность квантовой системы), коммутирующих между собой и с гамильтонианом.

Хорошо известно, что из-за нескольких технических нюансов это определение не может считаться полностью удовлетворительным [3]. Возможно, наиболее серьезной проблемой оказывается концепция функциональной независимости сохраняющихся величин, по крайней мере в том выделенном случае, когда первые интегралы являются самосопряженными. Действительно, согласно теореме фон Неймана [4] в этом случае как гамильтониан, так и его первые интегралы можно записать в виде функций другого самосопряженного оператора $A$ :

$$
H=f(A), \quad T_{i}=f_{i}(A),
$$

и, таким образом, функциональная независимость этих операторов невозможна. Эту трудность обычно преодолевают путем небольшой модифицикации концепции независимости, используемой в наивном определении квантовой интегрируемости. Например, можно считать, что квантовый гамильтониан $H$ и его первые интегралы $T_{i}$ являются хорошо определенными, гладкими функциями операторов координаты и импульса, т.е.

$$
H=g(\mathbf{X}, \mathbf{P}), \quad T_{i}=g_{i}(\mathbf{X}, \mathbf{P}),
$$

и требовать, чтобы функции $g_{i}$ были функционально независимыми. В литературе распространено много других модификаций наивной концепции квантовой интегрируемости. Например, определение квантовой интегрируемости, основанное на квазиклассическом пределе [5], достаточно часто используется в контексте квантового хаоса, особенно в математической литературе. Другая возможность состоит в том, чтобы выбрать некоторую реализацию гильбертова пространства, например, 
пространство $L^{2}\left(\mathbb{R}^{n}\right)$ квадратично-интегрируемых функций, и потребовать, чтобы первые интегралы были алгебраически независимыми дифференциальными операторами. Заметим, что, конечно, эти определения интегрируемости не являются полностью эквивалентными.

В настоящей работе мы используем аналогичную модифицикацию наивной концепции квантовой интегрируемости. Будем говорить, что оператор Гамильтона $H$ является квантово интегрируемым, если он обладает $n$ коммутирующими первыми интегралами $T_{i}$, которые могут быть преобразованы в гармонические осцилляторы следующим образом. Существует унитарный изоморфизм между гильбертовым пространством $\mathcal{H}$ гамильтониана $H$ и пространством квадратично-интегрируемых функций в евклидовом пространстве $\mathbb{R}^{n}$, который преобразует сохраняющиеся величины $T_{i}$ в операторы "чисел заполнения"

$$
N_{i}=\frac{1}{2}\left(P_{i}^{2}+X_{i}^{2}-1\right)=\frac{1}{2}\left(-\frac{\partial^{2}}{\partial x_{i}^{2}}+x_{i}^{2}-1\right),
$$

связанные с каждой координатой, а $H$ - в гладкую функцию операторов импульса и координаты, задаваемую каноническим квантованием интегрируемого классического гамильтониана. Обозначим этот квантовый гамильтониан через $H(\mathbf{X}, \mathbf{P})$, а его классический аналог - через $H(\mathbf{x}, \mathbf{p})$. Мы выбрали единицы, в которых $\hbar=2 m=1$. Напомним, что операторы координаты и импульса действуют в $L^{2}\left(\mathbb{R}^{n}\right)$ следующим образом:

$$
\mathbf{X} \psi(\mathbf{x})=\mathbf{x} \psi(\mathbf{x}), \quad \mathbf{P} \psi(\mathbf{x})=-i \boldsymbol{\nabla} \psi(\mathbf{x})
$$

В более физических терминах это определение означает, что гамильтониан является интегрируемым, если существует ортонормированный базис в гильбертовом пространстве, в котором этот гамильтониан представляет собой каноническое квантование $H(\mathbf{X}, \mathbf{P})$ интегрируемого классического гамильтониана $H(\mathbf{x}, \mathbf{p})$, где $\mathbf{x}$ и $\mathbf{p}$ принимают значения в евклидовом пространстве $\mathbb{R}^{n}$. Более того, этот гамильтониан можно рассматривать как нормальную форму Биркгоффа, т.е. $H(\mathbf{x}, \mathbf{p})=$ $f\left(x_{1}^{2}+p_{1}^{2}, \ldots, x_{n}^{2}+p_{n}^{2}\right)$. Конечно, $H$ и $H(\mathbf{X}, \mathbf{P})$ физически эквивалентны, поскольку они связаны унитарным преобразованием. Аналогичные определения интегрируемости и переменных действие-угол ранее появлялись в литературе в контексте конечномерной спиновой динамики [6].

В настоящей работе мы будем использовать введенное выше понятие квантовой интегрируемости, а для классической интегрируемости будем использовать определение Лиувилля. Возможно, сила введенного выше определения квантовой интегрируемости состоит в том, что оно распространяет алгебраические аспекты интегрируемости классической механики на квантовый случай, как показывает следующее отступление.

Отметим, что собственные функции оператора $H(\mathbf{X}, \mathbf{P})$ являются также собственными функциями каждого оператора $N_{i}$, поскольку он коммутирует с гамильтонианом $H(\mathbf{X}, \mathbf{P})$. Таким образом, нетрудно видеть, что каждая собственная функция 
$H(\mathbf{X}, \mathbf{P})$ факторизуется:

$$
\psi(\mathbf{x})=\prod_{i=1}^{n} \psi_{i}\left(x_{i}\right),
$$

где каждая $\psi_{i}$ - нормируемая собственная функция одномерного гармонического осциллятора, т.е.

$$
-\frac{d^{2} \psi_{i}(x)}{d x^{2}}+x^{2} \psi_{i}(x)=\left(2 n_{i}+1\right) \psi_{i}(x)
$$

Важно отметить, что для такой факторизации гамильтониан системы необходимо записать в подходящем базисе.

Можно проверить, что алгебраические аспекты определения квантовой интегрируемости, используемые в настоящей работе, весьма близки к лиувиллевским свойствам интегрируемости в классической механике. В рамках классической механики существует локальная замена координат (т.е. переменных действие-угол), преобразующая динамику системы в линейные потоки на торах (т.е. в невзаимодействующие гармонические осцилляторы), которая не может быть вычислена алгоритмически. В квантовом случае замена ортонормированного базиса, которая также не может быть получена с использованием алгоритмических процедур, преобразует динамику системы в динамику невзаимодействующих гармонических осцилляторов. Это показывают уравнения (2) и (3). Заметим, что в обоих случаях данное преобразование связано с сепарабельностью уравнения Гамильтона-Якоби ("в виде суммы”) в классическом случае и в смысле уравнений в частных производных ("в виде произведения") в квантовом.

Из этого отождествления следует, что операторы чисел заполнения $N_{i}$ являются квантовыми аналогами переменных действия в классической механике, и это хорошо известно. Таким образом, выражение оператора $H$ в терминах операторов $N_{i}$ посредством унитарного преобразования эквивалентно выражению квантового гамильтониана в терминах квантовых переменных действие-угол. Ясно, что $N_{i}$ представляют собой каноническое квантование переменных действия $x_{i}^{2}+p_{i}^{2}$ классического гамильтониана $H(\mathbf{x}, \mathbf{p})$; но также ясно, что сам гамильтониан $H$ не может быть получен как каноническое квантование этого классического гамильтониана. Действительно, если $H$ имеет классический аналог, из существования унитарного преобразования, выражающего $H$ в терминах операторов $N_{i}$, не следует, что существует каноническое преобразование, выражающее классический аналог $H$ в терминах его переменных действия. На самом деле, эти переменные действие-угол, возможно, не существуют для классического аналога $H$, как будет видно ниже, поскольку не имеется общего соответствия между классическими каноническими преобразованиями и квантовыми унитарными изоморфизмами. Для одномерных систем этот вопрос рассматривается в [7].

Работа построена следующим образом. В разделе 2 обсуждается достаточное условие интегрируемости, основанное на спектральных свойствах гамильтониана, а не на его симметриях. А именно гамильтониан без состояний рассеяния является 
интегрируемым независимо от размерности системы. Это условие позволяет доказать, что отсутствие универсальности положения классической интегрируемости не распространяется mutatis mutandis на квантовый случай. Мы усиливаем частные результаты, полученные Креханом [8] и Вайгертом [3], и рассматриваем несколько конкретных примеров. В разделе 3 обсуждается статистическое распределение нормированных разностей энергий для гамильтонианов без состояний рассеяния и появление статистики Пуассона. Поскольку эти гамильтонианы являются интегрируемыми, мы получаем дополнительное подтверждение гипотезы Берри-Табора без использования квазиклассического предела. Этот подход следует подходу Реланьо с соавторами [9], однако проверка, которую мы выполняем, не является численной. Нами получено также подтверждение того, что найденные этими авторами исключения из гипотезы Берри-Табора не являются общими случаями. Наша работа завершается некоторыми финальными замечаниями, представленными в разделе 4 .

\section{2. ИНТЕГРИРУЕМОСТЬ ГАМИЛЬТОНИАНОВ БЕЗ СОСТОЯНИЙ РАССЕЯНИЯ}

2.1. Основной результат. Для начала напомним некоторые положения, следуя определениям работы [10]. Необходимые понятия из спектральной теории будут сведены к минимуму для упрощения изложения.

Хорошо известно, что имеет место ортогональное разложение гильбертова пространства $\mathcal{H}$ гамильтониана $H$ в прямую сумму (замкнутых линейных оболочек) его связанных состояний $\mathcal{H}_{\mathrm{pp}}$ и состояний рассеяния $\mathcal{H}_{\text {cont }}$. Напомним, что интерпретация собственных функций гамильтониана как связанных состояний, а функций, соответствующих непрерывному спектру, - как состояний рассеяния, происходит из физически значимого положения отсутствия сингулярного непрерывного спектра [11]. Для простоты мы будем пользоваться терминами “состояния рассеяния" и "состояния в $\mathcal{H}_{\text {cont }}$ " как синонимами.

Разложение гамильтониана $H$ приводит к разложению его спектра $\sigma(H)$ на замыкание множества $\sigma_{\mathrm{pp}}(H)$ энергий связанных состояний гамильтониана, которое в большинстве случаев счетно, и множество $\sigma_{\text {cont }}(H)$ энергий, соответствующих состояниям рассеяния:

$$
\sigma(H)=\overline{\sigma_{\mathrm{pp}}(H)} \cup \sigma_{\text {cont }}(H) .
$$

Если гамильтониан $H$ не имеет состояний рассеяния (т.е. его непрерывный спектр $\sigma_{\text {cont }}(H)$ пуст), спектр гамильтониана $H$ представляет собой замыкание его собственных энергий. Тогда говорят, что $H$ должен иметь чисто точечный спектр, хотя это название может вводить в заблуждение, поскольку спектр может на самом деле включать произвольное число точек сгущения или замкнутых интервалов на вещественной прямой. Например, возможны спектры типа $\sigma(H)=[-10,-8] \cup[-5,-3] \cup$ $\left\{i^{-2}\right\}_{i=1}^{\infty}$, также являющиеся чисто точечными. В частности, неверно, что такие гамильтонианы имеют дискретный спектр или что их спектр может сгущаться только 
на бесконечности. Актуальные физические примеры гамильтонианов, демонстрирующих такое поведение, можно найти в работах [12]. Эти операторы описывают слабые возмущениия эффекта Штарка и фазовый переход (металл-изолятор) в модели Андерсона. Также известно [13], что можно построить такие гамильтонианы (возможно, с непрерывной частью спектра), что их точечные спектры будут плотны в любом подмножестве $\mathbb{R}$.

Следует напомнить, однако, что важным примером гамильтонианов без состояний рассеяния являются гамильтонианы с дискретным спектром. Этот случай наиболее интересен для приложений, хотя, конечно, не является общей ситуацией.

Для упрощения изложения мы предположим, что не существуют бесконечно вырожденные собственные энергии, как, конечно, случается в каждом физически интересном случае. Важно подчеркнуть, однако, что приемы, которые мы будем использовать для доказательства основных результатов, можно легко модифицировать для случая бесконечного вырождения.

Основной результат, который мы докажем в этом разделе, заключается в том, что каждый гамильтониан $H$ с чисто точечным спектром независимо от размерности $n$ системы является интегрируемым. Положим, что $\left\{E_{i}\right\}_{i=0}^{\infty}-$ последовательность собственных значений $H$, где каждая энергия входит столько раз, какова ее кратность, и что $\mathcal{H}$ - гильбертово пространство системы. Построим унитарный изоморфизм между $\mathcal{H}$ и пространством $L^{2}\left(\mathbb{R}^{n}\right)$ квадратично-интегрируемых функций на $\mathbb{R}^{n}$, приводящий $H$ к виду $H(\mathbf{X}, \mathbf{P})$, в котором его интегрируемость очевидна. Поскольку $L^{2}\left(\mathbb{R}^{n}\right)$ изоморфно $\mathcal{H}$, это можно понимать как замену ортонормированного базиса в $\mathcal{H}$.

Наш подход основан на реконструкции спектра гамильтониана $H$ с использованием гармонических осцилляторов. Насколько нам известно, эта идея восходит к Вайгерту [3], который использовал ее для того, чтобы указать на несколько недостатков наивного определения квантовой интегрируемости, как мы отмечали в разделе 1. Позже Крехан [8] использовал аналогичный подход для получения более явных решений похожей задачи. Основная сложность его конструкции заключалась в том, что требовалось ввести некоторый закон роста уровней энергии, для того чтобы гарантировать сходимость метода. Более того, для интерполяции последовательности энергий с использованием целых функций пришлось неявно предположить, что спектр гамильтониана $H$ не имеет конечных точек сгущения. Наш подход снимает оба эти предположения. Мы используем основанное на функциональном анализе построение с меньшими ограничениями, позволяющее использовать более широкий класс интерполирующих функций. Снятие этих ограничений играет существенную роль при изучении некоторых общих свойств гамильтонианов без состояний рассеяния, что и будет проделано в следующих разделах.

Напомним, что $\mathbf{X}$ и $\mathbf{P}$ - это операторы координаты и импульса на $L^{2}\left(\mathbb{R}^{n}\right)$ и рассмотрим оператор $N_{i}$ из уравнения (1). Ясно, что операторы $N_{i}$ коммутируют между собой, поскольку они действуют на различные координаты, их спектры 
являются чисто точечными и задаются множеством неотрицательных целых чисел $\mathbb{N}_{0}=\{0,1,2, \ldots\}$.

Пусть $\phi$ - произвольное биективное отображение $\mathbb{N}_{0}^{n}=\underbrace{\mathbb{N}_{0} \times \cdots \times \mathbb{N}_{0}}_{n \text { раз }}$ на $\mathbb{N}_{0}$. Тогда всегда можно выбрать гладкую функцию $f$, отображающую $\mathbb{R}^{n}$ в вещественные числа и удовлетворяющую уравнению

$$
f(\mathbf{n})=E_{\phi(\mathbf{n})}
$$

для всех $\mathbf{n}=\left(n_{1}, \ldots, n_{n}\right) \in \mathbb{N}_{0}^{n}$. Детали этого построения даны в приложении. Такая интерполирующая функция $f$, которую на самом деле можно представить как сходящийся степенной ряд, коль скоро последовательность собственных значений не имеет точек сгущения, позволяет определить гамильтониан

$$
H(\mathbf{X}, \mathbf{P})=f\left(N_{1}, \ldots, N_{n}\right)
$$

Это легко проделать, используя методы функционального анализа.

Гамильтониан (5) является интегрируемым. Чтобы это показать, заметим, что $H(\mathbf{X}, \mathbf{P})$ можно получить из интегрируемого классического гамильтониана

$$
H(\mathbf{x}, \mathbf{p})=f\left(\frac{1}{2}\left(x_{1}^{2}+p_{1}^{2}-1\right), \ldots, \frac{1}{2}\left(x_{n}^{2}+p_{n}^{2}-1\right)\right)
$$

посредством канонического квантования $(\mathbf{x} \mapsto \mathbf{X}, \mathbf{p} \mapsto \mathbf{P}=-i \boldsymbol{\nabla})$. Классический гамильтониан $H(\mathbf{x}, \mathbf{p})$ возникает по построению в нормальной форме Биркгоффа, его нормальные координаты являются глобальными и принимают значения в $\mathbb{R}^{2 n}$. Сохраняющиеся величины гамильтониана (5) можно рассматривать как операторы чисел заполнения, откуда следует интегрируемость $H(\mathbf{X}, \mathbf{P})$.

Заметим, что любая гладкая функция $f$, удовлетворяющая уравнению (4), порождает тот же квантовый гамильтониан, однако классический гамильтониан (6), определяемый этой функцией, зависит от выбора $f$. Поэтому мы имеем несчетное семейство классически интегрируемых гамильтонианов, все орбиты которых являются ограниченными и, как правило, плотными на торах, но каждый гамильтониан из этого семейства приводит к одному и тому же квантовому гамильтониану с чисто точечным спектром.

Теперь докажем, что существует такое унитарное преобразование $U$, отображающее $\mathcal{H}$ на $L^{2}\left(\mathbb{R}^{n}\right)$, что $H(\mathbf{X}, \mathbf{P})$ представляет собой выражение для $H$ в данной реализации гильбертова пространства, т.е. $H(\mathbf{X}, \mathbf{P})=U U^{\dagger}$. Отсюда следует, что $H$ коммутирует с $T_{i}=U^{\dagger} N_{i} U$, и доказательство интегрируемости $H$ завершается.

Нетрудно понять, что спектр $H(\mathbf{X}, \mathbf{P})$ (с учетом вырождений) совпадает со спектром $H$. Чтобы это показать, достаточно заметить, что, как обсуждалось в разделе 1 , собственные функции $H(\mathbf{X}, \mathbf{P})$ должны иметь вид произведения решений одномерных гармонических осцилляторов (2), а потому последовательность собственных значений $H(\mathbf{X}, \mathbf{P})$ с учетом вырождений задается выражением

$$
\sigma_{\mathrm{pp}}(H(\mathbf{X}, \mathbf{P}))=\{f(\mathbf{n})\}_{\mathbf{n} \in \mathbb{N}_{0}^{n}}=\left\{E_{i}\right\}_{i=0}^{\infty} .
$$


По построению $H(\mathbf{X}, \mathbf{P})$ не имеет непрерывного спектра, и поэтому его спектр есть замыкание множества его собственных значений, т.е.

$$
\sigma(H(\mathbf{X}, \mathbf{P}))=\overline{\sigma_{\mathrm{pp}}(H)}=\overline{\left\{E_{i}\right\}_{i=0}^{\infty}} .
$$

Поскольку $H$ не имеет состояний рассеяния, можно выбрать ортонормированный собственный базис $\left\{\phi_{i}\right\}_{i=0}^{\infty}$ в гильбертовом пространстве $\mathcal{H}$, где $\phi_{i}-$ собственная функция гамильтониана $H$ с энергией $E_{i}$. Поскольку $H(\mathbf{X}, \mathbf{P})$ также не имеет ни одного состояния рассеяния, можно найти такой ортонормированный базис в гильбертовом пространстве $L^{2}\left(\mathbb{R}^{n}\right)$ (мы обозначим его как $\left.\left\{\varphi_{i}\right\}_{i=0}^{\infty}\right)$, что $\varphi_{i}$ является собственной функцией $H(\mathbf{X}, \mathbf{P})$ с энергией $E_{i}$. Если теперь определить унитарное преобразование $U$, полагая

$$
U \phi_{i}=\varphi_{i}
$$

для каждого $i=0,1, \ldots$, то по построению получим

$$
H(\mathbf{X}, \mathbf{P})=U H U^{\dagger}
$$

Этим завершается доказательство утверждения.

2.2. Общие свойства квантовой интегрируемости. Физический интерес к критерию достаточной интегрируемости, обсуждавшемуся в предыдущем разделе, объясняется тем, что он дает точную информацию об интегрируемости некоторых квантовых систем, не опираясь на их конкретный вид. Этот факт можно использовать для доказательства некоторых свойств квантовой интегрируемости, являющихся аналогами известных результатов классической механики.

В первую очередь следует заметить, что из упомянутого выше критерия следует, что интегрируемые гамильтонианы являются плотными (в топологии нормы резольвенты), т.е. что любой заданный гамильтониан $H$ можно аппроксимировать интегрируемым гамильтонианом с точностью до произвольно малой ошибки. Это вытекает из того, что $H$ можно аппроксимировать гамильтонианами без состояний рассеяния с произвольной точностью (что эквивалентно приближению квадратичноинтегрируемых функций кусочно-постоянными функциями), и эти гамильтонианы являются интегрируемыми согласно основному результату. Следует отметить следующий факт: хотя мы установили, что интегрируемые гамильтонианы являются плотными (т.е. их топологически много), мы не можем гарантировать, что неинтегрируемых гамильтонианов мало в каком бы то ни было разумном смысле.

Таким образом, мы имеем существенное отличие от классического случая. Хорошо известно, что в классической механике имеется мало интегрируемых гамильтонианов, и действительно, Маркус и Майер [14] доказали, что "почти все" гамильтоновы системы (т.е. открытые и плотные подмножества в сильной $C^{\infty}$-топологии) являются неинтегрируемыми, поскольку классические интегрируемые гамильтонианы являются нигде не плотными. Конструкция Маркуса и Майера основана на невозможности получения локальных координат действие-угол для "почти всех" 
гамильтонианов. Как мы видели, на квантовый вариант этой локальной замены координат (а именно на унитарное преобразование $U$ ) определенно нет ограничений.

Заметим, что основной результат относится к абстрактным гамильтонианам и не ограничивается простейшим случаем операторов Шредингера, действующих в пространстве квадратично-интегрируемых функций. Исследование абстрактного случая предложено авторами работы [14] - им требовалось рассмотреть общие гамильтонианы, чтобы получить существенные результаты о повсеместности неинтегрируемости, а случай естественных гамильтонианов остался пока открытым. Замечательно, однако, что им удалось получить результаты о повсеместности неэргодичности в классической механике для естественных гамильтонианов.

Другим свойством интегрируемых гамильтонианов, которое можно вывести из основного результата, является то, что спектр интегрируемой квантовой системы вовсе не должен быть простым. Это противоречит общему мнению, обычно связывающему интегрируемые гамильтонианы с операторами, имеющими простые спектры, т.е. регулярно расположенные собственные значения, сгущающиеся в нуле, и непрерывный спектр выше нуля, как в случае атома водорода.

Покажем это. Пусть $C$ - любое замкнутое подмножество вещественных чисел, а $\left\{E_{i}\right\}_{i=0}^{\infty}-$ плотная последовательность в $C$, которая, как известно, существует. Построение $H(\mathbf{X}, \mathbf{P})$, приведенное в доказательстве основного результата, позволяет получить интегрируемый гамильтониан, спектр которого есть замыкание этой последовательности (см. формулу (7)), т.е. $C$ по определению. Отсюда мы получаем удивительное с физической точки зрения заключение, что имеются интегрируемые гамильтонианы, спектр которых есть, например, канторово множество.

Основной результат показывает, что гамильтониан $H$, спектр которого совпадает с мнимыми частями нулей дзета-функции Римана на критической прямой, должен быть интегрируемым, поскольку известно, что множество нулей является счетным. Это усиливает результат Крехана [8], который доказал, что существует интегрируемый квантовый гамильтониан, имеющий эту последовательность в качестве спектра. Напомним, что этот вопрос возник в контексте квантового хаоса, поскольку известно, что гамильтониан, имеющий эту последовательность в качестве спектра, подчиняется статистике гауссова унитарного ансамбля (GUE) случайных матриц, а эта статистика обычно связана с квантовым хаосом. Как мы покажем в следующем разделе, из этих результатов не следует, что классическое дополнение $H$ должно быть интегрируемым, и, следовательно, этот результат не запрещает появление неинтегрируемости в рамках классического предела или при использовании определения интегрируемости, основанного на квазиклассических методах.

Интересное практическое применение состоит в том, что существует, однако, (не единственный) интегрируемый классический гамильтониан $H(\mathbf{x}, \mathbf{p})$ вида $(6)$, квантовый аналог которого имеет мнимые части нулей дзета-функции Римана на критической прямой (если верить в гипотезу Римана) в качестве своего спектра. Это является исключением из утверждения Берри [15], согласно которому эти классиче-

4 Теоретическая и математическая физика, т. 148, № 2, 2006 г. 
ские гамильтонианы должны быть хаотическими. Тем не менее утверждение Берри в общем случае несомненно выполняется, и на это указывают результаты следующего раздела.

Напомним другой пример, показывающий, что существуют исключения из того, что статистика Вигнера-Дайсона характеризует неинтегрируемые классические системы. Он принадлежит Беннету с соавторами [16], которые построили ансамбль двумерных интегрируемых квантовых гамильтонианов, имеющих статистику гауссова ортогонального ансамбля (GOE) или статистику GUE при всех значениях параметров. В разделе 3 мы покажем, однако, что это явление не описывает общий случай, и, тем самым, не противоречит гипотезе Берри-Табора.

2.3. Приложения. Общеизвестно, что классическая интегрируемость не следует из квантовой интегрируемости. Мы можем это строго установить, используя наш основной результат. А впечатляющее эвристическое доказательство заключается в том, что интегрируемые гамильтонианы являются плотными в квантовом случае, тогда как в классическом случае они являются нигде не плотными.

Рассмотрим теперь классическую и квантовую интегрируемость нескольких актуальных систем. Наша цель двояка. С одной стороны, мы получим конкретные примеры гамильтонианов, которые интегрируемы как квантовые операторы, но классические аналоги которых неинтегрируемы. С другой стороны, мы получим нетривиальные примеры интегрируемых квантовых гамильтонианов, описывающих широкий класс физически интересных ситуаций, спектры которых могут оказаться заслуживающими изучения численными методами в контексте квантового хаоса.

В первую очередь рассмотрим движение свободной частицы на компактном римановом многообразии $M$. В квантовом случае динамика частицы задается гамильтонианом $H=-\Delta$, т.е. оператором Лапласа со знаком минус на многообразии. Известно, что этот гамильтониан имеет дискретный спектр, поэтому движение свободной квантовой частицы на компактном римановом многообразии является интегрируемым. Заметим, что этот результат обобщает результат Матвеева и Топалова [17], которые доказали квантовую интегрируемость этого гамильтониана в частном случае многообразия, имеющего непропорциональные геодезически эквивалентные метрики. Однако в этом частном случае их результаты сильнее, поскольку дают некоторую информацию о виде сохраняющихся величин.

Наоборот, классическое движение свободной частицы на компактном римановом многообразии $M$ дается уравнением геодезических, которое обычно является неинтегрируемым. В частности, для случая, когда $M$ имеет отрицательную секционную кривизну, Аносов [18] доказал, что орбиты являются эргодическими, и поэтому гамильтоново векторное поле не может иметь непрерывных первых интегралов, отличных от энергии.

Другой интересный пример - это движение частицы в $n$-мерном евклидовом пространстве $\mathbb{R}^{n}$ под действием сильно связывающего потенциала $V$. Более точно, пусть потенциал ограничен снизу $(V(\mathbf{x}) \geqslant c)$ и стремится к бесконечности с ростом 
расстояния от начала отсчета $\left(\lim _{|\mathbf{x}| \rightarrow+\infty} V(\mathbf{x})=+\infty\right)$. В случае, когда $V$ удовлетворяет некоторым специальным мягким условиям, Браудер [19] доказал, что гамильтониан $H=-\Delta+V(\mathbf{x})$ не имеет состояний рассеяния, поэтому квантовое движение является интегрируемым. Тем не менее хорошо известно, что наличие сильно связывающего потенциала недостаточно для того, чтобы классический гамильтониан

$$
H(\mathbf{x}, \mathbf{p})=\mathbf{p}^{2}+V(\mathbf{x})
$$

был интегрируемым. Действительно, известны примеры [20], в которых выполняются все требования на потенциал $V$, однако гамильтониан (8) является неинтегрируемым в терминах мероморфных первых интегралов.

Двумерная модель Кронига-Пенни в присутствии магнитного поля, использовавшаяся для имитации кристаллических ионов в квантовых твердых телах, демонстрирует тот же эффект. Легко показать, что квантовый гамильтониан

$$
H=\left[-i \frac{\partial}{\partial x}+\frac{e B y}{2 c}\right]^{2}+\left[-i \frac{\partial}{\partial y}-\frac{e B x}{2 c}\right]^{2}+V_{0}\left(\cos \frac{2 \pi x}{a}+\cos \frac{2 \pi y}{a}\right)
$$

имеет дискретный спектр. В этой формуле $a$ - шаг решетки, $e$ - заряд электрона, $c$ - скорость света, $B$ - (постоянное) магнитное поле, а $V_{0}$ - положительная константа. Чтобы доказать, что описанный выше гамильтониан имеет дискретный спектр, достаточно заметить, что $H$ ограничен снизу гамильтонианом магнетика

$$
H_{0}=\left[-i \frac{\partial}{\partial x}+\frac{e B y}{2 c}\right]^{2}+\left[-i \frac{\partial}{\partial y}-\frac{e B x}{2 c}\right]^{2}-2 V_{0}
$$

который, как известно, имеет дискретный спектр, и применить принцип минимакса. Однако численные методы показали, что его классический вариант

$$
H(\mathbf{x}, \mathbf{p})=\left(p_{x}+\frac{e B y}{2 c}\right)^{2}+\left(p_{y}-\frac{e B x}{2 c}\right)^{2}+V_{0}\left(\cos \frac{2 \pi x}{a}+\cos \frac{2 \pi y}{a}\right)
$$

демонстрирует стандартные характерные свойства хаоса [21] и не проходит тест Пенлеве [22].

Стоит также упомянуть другой знаменитый гамильтониан, демонстрирующий аналогичное поведение. Рассмотрим движение частицы на плоскости в потенциале $V(\mathbf{x})=x^{2} y^{2}$, где $\mathbf{x}=(x, y)$. Легко видеть, что у классического гамильтониана

$$
H(\mathbf{x}, \mathbf{p})=\mathbf{p}^{2}+V(\mathbf{x})
$$

есть орбиты рассеяния, например, $y=0$. Однако Саймон [23] доказал, что его квантовый вариант

$$
H=-\Delta+V(\mathbf{x})
$$

не имеет состояний рассеяния. Это удивительное явление возникает вследствие того, что на самом деле гамильтониан (9) имеет очень мало направлений рассеяния. 
Интересно отметить, что другим любопытным свойством указанного потенциала является то, что, не имея состояний рассеяния, квантовый гамильтониан (10) является интегрируемым, тогда как прямое применение критерия Йошиды [24] показывает, что (9) таковым не является (по крайней мере при аналитических первых интегралах). Действительно, численные исследования этого классического гамильтониана показывают сложную структуру орбит.

Что касается применимости нашего основного результата, заметим, что у него также есть приложения в области решаемых квантовых моделей, особенно в таких ситуациях, когда легче проверить точную или квазиточную разрешимость модели, чем ее интегрируемость, а спектр модели дискретен. Такая ситуация возникает, например, при изучении спиновых цепочек Холдейна-Шастри, взаимодействующих с внешним полем, с использованием метода операторов Данкла [25].

Наконец, следует напомнить, что результаты Жанга с соавторами [26] утверждают, что из квантовой интегрируемости следует классическая интегрируемость, но их определение квантовой интегрируемости слабее, чем то, которое мы используем в настоящей работе. Причина этого кажущегося противоречия заключается в том, что Жанг с соавторами не указали никакой степени регулярности классических первых интегралов, например, гладкости или по крайней мере непрерывности. Внимательный взгляд на доказательство нашего основного результата показывает, что нарушение интегрируемости классического аналога гамильтониана $H$ связано с тем, что унитарное преобразование $U$, отображающее гильбертово пространство $\mathcal{H}$ в пространство квадратично-интегрируемых функций на $\mathbb{R}^{n}$, не индуцирует симплектоморфизм из фазового пространства системы в $\mathbb{R}^{2 n}$. Интересно заметить, что и симплектоморфизм в общем случае не приводит к унитарному преобразованию [27].

\section{3. СТАТИСТИЧЕСКОЕ РАСПРЕДЕЛЕНИЕ УРОВНЕЙ ЭНЕРГИИ}

Исследование статистического распределения энергий в интегрируемых и неинтегрируемых системах (с различными определениями интегрируемости) представляет собой привлекательную область современной квантовой физики. Одним из поворотных пунктов в этой области является так называемая гипотеза Берри-Табора, которая устанавливает связь между распределением энергий для данного гамильтониана и его интегрируемостью, причем используемое определение интегрируемости основанно на квазиклассическом приближении.

Содержание гипотезы Берри-Табора заключается в том, что если $H$ является каноническим квантованием интегрируемого классического гамильтониана $H(\mathbf{x}, \mathbf{p})$, то статистическое распределение разности его нормированных энергий является пуассоновым. Таким образом, статистическое распределение энергий связано с хорошо определенным и математически точным квазиклассическим подходом к интегрируемости. Берри и Табор [28] дали полустрогое доказательство гипотезы, носящей их имена, и имеются многочисленные подтверждения верности их утверждения [29]. 
Эта гипотеза была недавно строго доказана в частном случае частицы, движущейся на торе в присутствии однородного магнитного поля [30].

Актуальным для исследований вопросом является изучение статистических свойств энергетического спектра квантовых интегрируемых систем (возможно, вообще не имеющих классических аналогов) без использования квазиклассического предела. Например, Реланьо с соавторами [9] для того, чтобы провести численную проверку гипотезы Берри-Табора, использовали точно решаемые модели РичардсонаГодена, квантовая интегрируемость которых установлена без обращения к квазиклассическому пределу. Было найдено несколько исключений из гипотезы БерриТабора, однако с помощью численных методов эти авторы показали, что небольшие интегрируемые возмущения восстанавливают спектр Пуассона.

Утверждение данного раздела состоит в том, что гамильтонианы, имеющие пуассоново распределение энергий, плотны в ансамбле гамильтонианов с чисто точечным спектром. Прежде чем это доказывать, следует начать с некоторых замечаний.

В первую очередь отметим, что, конечно, данное утверждение не противоречит общепринятому в теории квантового хаоса мнению о том, что в классе всех гамильтонианов с хорошо определенным классическим пределом те из них, которые не имеют пуассонова распределения энергий и чьи классические аналоги являются неинтегрируемыми, являются в некотором смысле "случаями общего положения". Из нашего утверждения не следует, что не имеющих пуассонова распределения энергий неинтегрируемых квантовых гамильтонианов или гамильтонианов с неинтегрируемым классическим пределом мало (можно вспомнить аналогичный случай рациональных и вещественных чисел), поскольку общепринятое мнение состоит в том, что они должны значительно превосходить числом интегрируемые. Более того, наш подход не привлекает классический предел.

Утверждение этого раздела можно применить, для того чтобы показать, не прибегая к численным расчетам, почему существование интегрируемых моделей, реализующих хаотические спектры, показанное в работах Бенета с соавторами [16] и Реланьо с соавторами [9], не имеет отношения к гипотезе Берри-Табора. Действительно, пусть $H$ - гамильтониан Ричардсона-Годена, параметры которого приводят к распределению Вигнера, или гамильтониан, принадлежащий ансамблю, который рассматривали Бенет с соавторами. В обоих случаях $H$ имеет чисто точечный спектр, и из нашего утверждения следует, что гамильтонианы, имеющие пуассоново распределение энергий, плотны в множестве гамильтонианов без состояний рассеяния и являются интегрируемыми согласно нашему основному результату. Поэтому существует такое произвольно малое возмущение, сохраняющее интегрируемость $H$ (хотя, возможно, не в пределах того же семейства, как это следует из результатов, полученных Беннетом с соавторами), что статистическое распределение энергий быстро распадается и принимает вид распределения Пуассона.

Теперь докажем утверждение. Поскольку доказательство достаточно длинное, мы поделим его на четыре этапа. Чтобы избавиться от несущественных степеней 
свободы, удобно отождествлять гамильтонианы, отличающиеся только заменой ортонормированного базиса.

Шаг 1. Сначала обозначим через $\mathcal{A}_{\mathrm{pp}}$ множество унитарных классов гамильтонианов размерности $n$ с чисто точечным спектром, т.е. множество гамильтонианов без состояний рассеяния, определенных с точностью до замены ортонормированного базиса. Унитарный класс, определяемый гамильтонианом $H$, обозначим через $[H]$. Пусть $\mathcal{C}$ - множество последовательностей (возможно, повторяющихся) вещественных чисел,

$$
\mathcal{C}=\left\{\left\{c_{i}\right\}_{i=0}^{\infty}: c_{i} \in \mathbb{R}\right\}
$$

где две последовательности, отличающиеся только порядком своих элементов, считаются тождественными.

Можно видеть, что унитарные классы гамильтонианов в $\mathcal{A}_{\mathrm{pp}}$ и последовательности вещественных чисел в $\mathcal{C}$ находятся во взаимно однозначном соответствии. Для доказательства этого факта заметим, что каждый класс $[H] \in \mathcal{A}_{\mathrm{pp}}$ порождает последовательность своих собственных значений $\left\{E_{i}\right\}$, с точностью до упорядочения. И обратно, неупорядоченная последовательность $\left\{E_{i}\right\}$ единственным образом определяет класс $[H] \in \mathcal{A}_{\mathrm{pp}}$, спектром которого является эта последовательность. Это показывает следующее элементарное рассуждение. Если $\left\{\varphi_{i}\right\}_{i=0}^{\infty}-$ ортонормированный базис в гильбертовом пространстве, можно определить представитель $H \in[H]$ как

$$
H=\sum_{i=0}^{\infty} E_{i}\left|\varphi_{i}\right\rangle\left\langle\varphi_{i}\right| .
$$

Кроме того, любой другой гамильтониан в $\mathcal{A}_{\mathrm{pp}}$, реализующий те же собственные энергии,

$$
\widetilde{H}=\sum_{i=0}^{\infty} E_{i}\left|\tilde{\varphi}_{i}\right\rangle\left\langle\tilde{\varphi}_{i}\right|,
$$

где $\left\{\tilde{\varphi}_{i}\right\}_{i=0}^{\infty}-$ другой ортонормированный базис, эквивалентен $H$ вследствие существования унитарного преобразования $U \tilde{\varphi}_{i}=\varphi_{i}$, которое преобразует нормированные собственные функции гамильтониана $\widetilde{H}$ в нормированные собственные функции гамильтониана $H$. Таким образом, неупорядоченная последовательность $\left\{E_{i}\right\} \in \mathcal{C}$ определяет единственный класс $[H] \in \mathcal{A}_{\mathrm{pp}}$, который имеет эту последовательность в качестве своего спектра.

Шаг 2. Взаимно однозначное соответствие между $\mathcal{A}_{\mathrm{pp}}$ и $\mathcal{C}$ можно продолжить до топологической эквивалентности. В дальнейшем мы используем этот факт для ответа на вопрос, является ли заданное множество гамильтонианов плотным в $\mathcal{A}_{\mathrm{pp}}$ или $\mathcal{C}$.

Снабдим $\mathcal{C}=\mathbb{R}^{\mathbb{N}_{0}}$ топологией произведения, так что $\left\{c_{i}^{(n)}\right\}$ сходится к $\left\{c_{i}\right\}$, если и только если $c_{i}^{(n)}$ сходится к $c_{i}$ для всех $i$. Тогда взаимно однозначное соответствие между $\mathcal{A}_{\mathrm{pp}}$ и $\mathcal{C}$ топологизирует $\mathcal{A}_{\mathrm{pp}}$. Действительно, наследуемую топологию 
легко выразить в терминах обычной топологии нормы резольвенты, поскольку последовательность $\left\{\left[A_{n}\right]\right\} \subset \mathcal{A}_{\text {рр }}$ сходится к $[A]$, если и только если существуют такие представители $\tilde{A}_{n} \in\left[A_{n}\right], \tilde{A} \in[A]$, что $\tilde{A}_{n}$ сходится к $\tilde{A}$ в смысле нормы резольвенты. Достаточность существования сходящейся к $\tilde{A} \in[A]$ последовательности представителей для сходимости в $\mathcal{A}_{\mathrm{pp}}$ очевидна, необходимость получается получается путем выбора представителей $\tilde{A}_{n}, \tilde{A}$ с общим ортонормированным базисом собственных функций $\left\{\varphi_{i}\right\}$ и применения формулы

$$
\begin{aligned}
\left\|\left(\tilde{A}_{n}-\lambda\right)^{-1}-(\tilde{A}-\lambda)^{-1}\right\| & =\| \sum_{i=1}^{\infty}\left[\left(c_{i}^{(n)}-\lambda\right)^{-1}-\left(c_{i}-\lambda\right)^{-1}\right]\left|\varphi_{i}\right\rangle\left\langle\varphi_{i}\right| \|= \\
& =\sup _{i \in \mathbb{N}_{0}}\left|\frac{1}{c_{i}^{(n)}-\lambda}-\frac{1}{c_{i}-\lambda}\right|,
\end{aligned}
$$

которая выполняется для любых $\lambda \in \mathbb{C}$ при $\operatorname{Im} \lambda \neq 0$. Таким образом, сходимость и плотность в $\mathcal{C}$ и $\mathcal{A}_{\mathrm{pp}}$ становятся эквивалентными.

Шаг 3. Теперь докажем, что множество гамильтонианов без состояний рассеяния (определяемых с точностью до унитарного преобразования), спектры которых имеют равномерное распределение, является плотным в указанной выше топологии.

Поскольку $\mathbb{R}$ не ограничено, необходимо рассматривать такие взвешенные меры $w(x) d x$ (где весовая функция $w$ положительна, непрерывна и удовлетворяет соотношению $\left.\int_{-\infty}^{+\infty} w(x) d x=1\right)$, чтобы мера всего $\mathbb{R}$ стала конечной [31], [32]. Напомним, что последовательность вещественных чисел $\left\{c_{i}\right\}$ имеет равномерное распределение относительно веса $w$, если

$$
\lim _{N \rightarrow \infty} \frac{1}{N} \sum_{i=0}^{N-1} f\left(c_{i}\right)=\int_{-\infty}^{+\infty} f(x) w(x) d x
$$

для любой непрерывной функции $f$ с компактным носителем. Заметим, что это определение также справедливо для неупорядоченных последовательностей, поскольку упомянутый выше предел (который существует и равен интегралу, стоящему в правой части) не зависят от упорядочения.

Теперь утверждение, сделанное на данном шаге, следует из теорем об эргодичности (см. [31] и теорему 2.4 в [33]), согласно которым равномерно распределенные относительно веса $w$ последовательности являются плотными в $\mathcal{C}$. Из рассуждений, проведенных на шаге 2 , следует, что унитарные классы в $\mathcal{A}_{\mathrm{pp}}$ с равномерно распределенными относительно веса $w$ спектрами также являются плотными. Поскольку это свойство верно для любой весовой функции $w$, далее мы будем ее опускать.

Шаг 4. Докажем, что интервалы между уровнями энергии в общем случае распределены по Пуассону, если собственные значения гамильтониана подчиняются равномерному распределению. Напомним, что имеются хорошо известные исключения, в которых из равномерного распределения энергий не следует с необходимостью, что разности энергий распределены по Пуассону. Примером такого явления служат многомерные гармонические осцилляторы [34]. К счастью, для почти 
всех последовательностей энергий $\left\{E_{i}\right\}$, при доказательстве факта распределения по Пуассону можно использовать следующее рассуждение из книги Гутцвиллера [35] (см. раздел 16.3), восходящее к работе Портера [36].

Для последовательностей энергий в общем положении соответствующее спектральное распределение $Q$ и интервалы между уровнями энергии $P$ хорошо определены. Пусть $Q(x) d x$ - вероятность того, что нормированная собственная энергия гамильтониана $H$ находится в интервале $(E+x, E+x+d x)$, где $E$ - нормированное собственное значение $H$. Аналогично, пусть $P(x) d x$ - вероятность нахождения двух последовательных нормированных уровней на расстоянии $x$.

Хорошо известно, что $P$ и $Q$ связаны уравнением

$$
P(x)=Q(x) \exp \left[-\int_{0}^{x} Q(\xi) d \xi\right],
$$

которое было установлено Вигнером. Дадим для полноты доказательство уравнения (12). Пусть $g(x)$ - вероятность не обнаружить нормированный уровень на расстоянии меньше $x$ от другого. Тогда, очевидно, $g(0)=1$. Вероятность не обнаружить нормированный уровень на расстоянии меньше $x+d x$ можно выразить как

$$
g(x+d x)=g(x)(1-Q(x) d x),
$$

поскольку $g(x)$ - вероятность не обнаружить уровень на расстоянии меньше $x$, а $1-Q(x) d x$ - вероятность не обнаружить уровень на расстоянии $d$, где $x \leqslant d \leqslant x+d x$. Разлагая уравнение (13), находим

$$
\frac{d g(x)}{d x}=-g(x) Q(x)
$$

и, таким образом, $g(x)=\exp \left[-\int_{0}^{x} Q(\xi) d \xi\right]$. Окончательно получаем, что

$$
P(x) d x=g(x) Q(x) d x
$$

по определению, и поэтому уравнение (12) выполнено.

Равенство (12) показывает, что если энергии гамильтониана $H$ распределены равномерно, а потому $Q(x)=1$, то $P(x)=e^{-x}$, что мы и хотели доказать.

Итак, мы завершили доказательство того факта, что для плотного подмножества всех гамильтонианов без состояний рассеяния (определенных с точностью до замены ортонормированного базиса), которые являются интегрируемыми в силу основного результата п. 2.1, разности нормированных уровней энергии распределены по Пуассону.

Следует заметить, что определение интегрируемости, использованное в настоящей работе, и то, которое использовали Реланьо с соавторами, несколько отличаются от квазиклассического определения, которое использовали в своей работе Берри и Табор. Но результаты, полученные в данном разделе, как уже говорилось, дают дополнительное подтверждение этой прославленной гипотезы. 


\section{4. ЗАКЛЮЧИТЕЛЬНЫЕ ЗАМЕЧАНИЯ}

Мы получили достаточное условие интегрируемости квантового гамильтониана: он всегда интегрируем, коль скоро в нем нет состояний рассеяния. Насколько нам известно, такой спектральный подход к квантовой интегрируемости является новым в литературе и частично связывает различные подходы к интегрируемости, используемые в квантовой физике.

Мы также показали, что определение интегрируемости, используемое в настоящей работе, чрезвычайно удобно, поскольку оно наследует алгебраические свойства классической концепции интегрируемости Лиувилля. Мы полагаем, что детальное изучение соотношений между этим определением и квазиклассическим должно вызвать интерес.

В п. 2.2 было доказано, что вопреки интуиции, основанной на классической физике, имеется очень много интегрируемых квантовых систем. Было бы интересно узнать, справедлив ли этот результат и в квантовой теории поля, или же данное явление возникает только в квантовой механике. Некоторые авторы полагали, что квантовая механика очень часто оказывается интегрируемой, хотя рассматриваемым ими определениям интегрируемости до некоторой степени не хватало физической интерпретации, которая есть в наших определениях. Например, Купершмидт [37] доказал, что гамильтоновы уравнения движения имеют бесконечное множество сохраняющихся величин в смысле дифференциальных уравнений в частных производных, а Чирелли и Пиццокеро [38] развили интересное абстрактное определение интегрируемости в терминах инвариантных цилиндров в пространстве лучей.

Кроме того, нам удалось установить, что вопреки общепринятой точке зрения спектр интегрируемой системы не обязан являться простым. Отсюда можно предположить, что интересно было бы найти интегрируемые гамильтонианы (существование которых следует из нашего основного результата), реализующие канторово множество в качестве своего спектра, и выяснить, можно ли их переписать в виде оператора Шредингера. Напомним, что пример гамильтониана, спектр которого дается канторовым множеством, был найден Хофштадтером [39] в качестве эффективной однозонной модели электронного кристалла в однородном магнитном поле. Этот гамильтониан не возникает в естественной форме и, насколько нам известно, его интегрируемость никогда не исследовалась.

В п. 2.3 мы привели несколько приложений основного результата и несколько конкретных систем, которые интегрируемы в квантовом случае, но неинтегрируемы в классическом случае. Напомним, что приведенные примеры отвечают нескольким физически актуальным ситуациям, таким как частица в сильно связывающем потенциале или свободная частица на компактном многообразии.

Наконец, в п. 3, опираясь на гипотезу Берри-Табора и следуя более ранним работам по этой теме, мы исследовали статистическое распределение энергий гамильтонианов без состояний рассеяния. Мы строго получили, что плотное подмножество 
в этом ансамбле распределено по Пуассону, тем самым предоставив еще одно свидетельство справедливости этой прославленной гипотезы.

\section{ПРИЛОЖЕНИЕ}

Нетрудно привести явное выражение для гладкой интерполирующей функции $f$, используемой в формуле (4) в п. 2.1. Пусть $\phi: \mathbb{N}_{0}^{n} \rightarrow \mathbb{N}_{0}-$ произвольная биекция $\mathbb{N}_{0}^{n}$ на $\mathbb{N}_{0}$. Примером такой биекции является функция

$$
\phi_{\boldsymbol{\pi}}(\mathbf{n})=\operatorname{card}\left\{\mathbf{m} \in \mathbb{N}_{0}^{n}: \boldsymbol{\pi}^{\mathbf{m}}<\boldsymbol{\pi}^{\mathbf{n}}\right\}
$$

где использованы обозначения

$$
\boldsymbol{\pi}^{\mathbf{n}}=\pi_{1}^{n_{1}} \ldots \pi_{n}^{n_{n}}
$$

а $\pi_{1}, \ldots, \pi_{n}$ - взаимно простые положительные целые числа. Пусть теперь гладкая функция с горбом $\varphi: \mathbb{R} \rightarrow \mathbb{R}$ задается в виде

$$
\varphi(x)= \begin{cases}e^{-4 x^{2} /\left(1-4 x^{2}\right)}, & \text { если }|x| \leqslant 1 / 2, \\ 0, & \text { если }|x| \geqslant 1 / 2 .\end{cases}
$$

Определим функцию $f: \mathbb{R}^{n} \rightarrow \mathbb{R}$ как

$$
f(\mathbf{x})=\sum_{\mathbf{m} \in \mathbb{N}_{0}^{n}} E_{\phi(\mathbf{m})} \varphi(|\mathbf{x}-\mathbf{m}|) .
$$

Этот ряд сходится поточечно всюду, а также равномерно на каждом компактном множестве. Очевидно, кроме того, что эта функция бесконечно дифференцируема, и $f(\mathbf{n})=E_{\phi(\mathbf{n})}$. Тем самым утверждение установлено.

Если последовательность энергий не имеет точек сгущения (за исключением бесконечности), интерполирующую функцию $f$ можно выбрать аналитической и, следовательно, разложить в сходящийся степенной ряд. Чтобы построить эту аналитическую функцию, используем функции $W_{j}: \mathbb{C} \rightarrow \mathbb{C}$, определенные следующим равенством:

$$
W_{j}(z)=(1-z) \exp \left[\sum_{k=1}^{j} \frac{z^{k}}{k}\right],
$$

где $j \in \mathbb{N}_{0}$. Теперь можно определить функцию

$$
g(z)=\prod_{\mathbf{n} \in \mathbb{N}_{0}^{n}} W_{\phi(\mathbf{n})}\left(\frac{z}{\boldsymbol{\pi}^{\mathbf{n}}}\right),
$$

где положительное целое $\boldsymbol{\pi}^{\mathbf{n}}$ задается равенством (П.1). Поскольку множество $\left\{\boldsymbol{\pi}^{\mathbf{n}}\right\}_{\mathbf{n} \in \mathbb{N}_{0}^{n}}$ не сгущается ни в какой конечной точке и не содержит нуля, указанное произведение сходится и определяет аналитическую функцию на комплексной плоскости, единственные нули которой лежат в точках $z=\boldsymbol{\pi}^{\mathbf{n}} \quad\left(\mathbf{n} \in \mathbb{N}_{0}^{n}\right)[40]$. 
Пусть теперь $h: \mathbb{C} \rightarrow \mathbb{C}$ - функция Миттаг-Леффлера [40] с полюсами в $\left\{\boldsymbol{\pi}^{\mathbf{n}}\right\}_{\mathbf{n} \in \mathbb{N}_{0}^{n}}$, главная часть которой при каждом $z=\boldsymbol{\pi}^{\mathbf{n}}$ имеет вид

$$
P_{\mathbf{n}}(z)=\frac{E_{\phi(\mathbf{n})}}{W_{\phi(\mathbf{n})}^{\prime}\left(\boldsymbol{\pi}^{\mathbf{n}}\right)}\left(z-\boldsymbol{\pi}^{\mathbf{n}}\right)^{-1} .
$$

Заметим, что входящую в последнее выражение производную функции $W_{\phi(\mathbf{n})}$ в точке $\boldsymbol{\pi}^{\mathbf{n}}$ можно вычислить как

$$
W_{\phi(\mathbf{n})}^{\prime}\left(\boldsymbol{\pi}^{\mathbf{n}}\right)=e^{H_{\phi(\mathbf{n})}} \prod_{\mathbf{n} \neq \mathbf{m} \in \mathbb{N}_{0}^{n}} W_{\phi(\mathbf{m})}\left(\frac{\boldsymbol{\pi}^{\mathbf{n}}}{\boldsymbol{\pi}^{\mathbf{m}}}\right),
$$

где $H_{j}=\sum_{k=1}^{j} k^{-1}-j$-е гармоническое число.

Определим интерполирующую функцию $f: \mathbb{R}^{n} \rightarrow \mathbb{R}$ как $f(\mathbf{x})=g\left(\boldsymbol{\pi}^{\mathbf{x}}\right) h\left(\boldsymbol{\pi}^{\mathbf{x}}\right)$. Поскольку функция

$$
\boldsymbol{\pi}^{\mathbf{x}}=\pi_{1}^{x_{1}} \ldots \pi_{n}^{x_{n}}=\exp \left(\sum_{j=1}^{n} x_{j} \ln \pi_{j}\right)
$$

аналитична, очевидно, что такова же и $f$. При этом [40] для $f$ при всех $\mathbf{n} \in \mathbb{N}_{0}^{n}$ выполнено равенство $f(\mathbf{n})=E_{\phi(\mathbf{n})}$, и, таким образом, $f$ представляет собой искомую функцию.

Благодарности. Авторы благодарят профессоров Ф. Финкеля, А. ГонсалесаЛопеса и М. А. Родригеса за ценные предложения в ходе написания данной работы. А. Энсисо поддержан FPU Scholarship со стороны Испанского министерства образования, а также благодарен DGI за частичную финансовую поддержку в рамках гранта № BFM2002-02646.

\section{Список литературы}

[1] M. A. Olshanetsky, A. M. Perelomov, Phys. Rep., 94 (1983), 313; A. V. Turbiner, Commun. Math. Phys., 118 (1988), 467.

[2] G. Casati, F. Valz-Gris, I. Guarnieri, Lett. Nuovo Cimento, 28 (1980), 279; O. Bohigas, M. J. Giannoni, C. Schmit, Phys. Rev. Lett., 52 (1984), 1.

[3] S. Weigert, Phys. D, 56 (1992), 107.

[4] И. Нейман, Математические основы квантовой механики, Наука, М., 1964.

[5] Y. Colin de Verdière, Enseign. Math., 44 (1998), 23.

[6] S. Weigert, G. Müller, Chaos, Solitons \& Fractals, 5 (1995), 1419.

[7] M. Moshinsky, T. H. Seligman, Ann. Physics, 114 (1978), 243; J. Deenen, M. Moshinsky, T. H. Seligman, Ann. Phys., 127 (1980), 458.

[8] P. Crehan, J. Phys. A, 28 (1995), 6389.

[9] A. Relaño, J. Dukelsky, J. M. Gómez, J. Retamosa, Phys. Rev. E, 70 (2004), 026208.

[10] М. Рид, Б. Саймон, Методы современной математической физики, m. 1, Мир, М., 1977.

[11] W. Thirring, Quantum Mathematical Physics, Springer, Berlin, 2002.

[12] С.Н. Набоко, ТМФ, 68:1 (1986), 18; С.Н. Набоко, А.Б. Пушницкий, Функи. анализ и его прилож., 29:4 (1995), 31.

[13] B. Simon, Proc. Amer. Math. Soc., 125 (1997), 203.

[14] L. Markus, K. R. Meyer, Mem. Amer. Math. Soc., 144 (1974), 1. 
[15] M. V. Berry, Proc. R. Soc. London A, 400 (1985), 229.

[16] L. Benet, F. Leyvraz, T. H. Seligman, Phys. Rev. E, 68 (2003), 045201(R).

[17] V.S. Matveev, P. J. Topalov, Math. Z., 238 (2001), 833.

[18] Д. В. Аносов, Тр. МИАН, 90 (1967), 1.

[19] F.E. Browder, Math. Ann., 142 (1961), 22.

[20] M. E. Sansaturio, I. Yigo-Aguiar, J. M. Ferrándiz, J. Phys. A, 30 (1997), 5869.

[21] M. del Mar Espinosa, M. A. Martín-Delgado, A. Niella, D. Páramo, J. Rodríguez-Laguna, Chaos in the classical analogue of the Hofstadter problem, chao-dyn/9808012.

[22] A. Ramani, B. Grammaticos, T. Bountis, Phys. Rep., 180:3 (1989), 159.

[23] B. Simon, Ann. Phys., 146 (1983), 209.

[24] H. Yoshida, Phys. D, 29 (1987), 128.

[25] A. Enciso, F. Finkel, A. González-López, M. A. Rodríguez, Nucl. Phys. B, 707 (2005), 553; hep-th/0406054; J. Nonlin. Math. Phys., 12 (2003), 253; hep-th/0412098.

[26] W. M. Zhang, D. H. Feng, J.-M. Yuan, S.-J. Wang, Phys. Rev. A, 40 (1989), 438.

[27] L. Kaplan, New J. Phys., 4 (2002), 90.

[28] M. V. Berry, M. Tabor, Proc. R. Soc. Lond. A, 356 (1977), 375.

[29] M. Razavy, Phys. Lett. A, 113 (1985), 297; T. H. Seligman, J. J. Verbaarschot, Phys. Rev. Lett., 56 (1986), 2767.

[30] J. Marklof, Ann. of Math., 158 (2003), 419.

[31] G. Helmberg, Math. Z., 86 (1964), 157.

[32] J. Lesca, Enseign. Math., 17 (1971), 311.

[33] L. Kuipers, H. Niederreiter, Uniform Distribution of Sequences, Wiley, N.Y., 1974.

[34] A. Pandey, R. Ramaswamy, Phys. Rev. A, 43 (1991), 4237.

[35] M. C. Gutzwiller, Chaos in Classical and Quantum Mechanics, Springer, N.Y., 1990.

[36] C. F. Porter, Statistical Theory of Spectra: Fluctuations, Academic Press, London, 1965.

[37] B. A. Kupershmidt, Phys. Lett. A, 109 (1985), 136.

[38] R. Cirelli, L. Pizzocchero, Nonlinearity, 3 (1990), 1057.

[39] D. R. Hofstadter, Phys. Rev. B, 14 (1976), 2239.

[40] W. Rudin, Real and Complex Analysis, McGraw-Hill, N.Y., 1966.

Поступила в редакцию 28.09.2005 